\title{
Stochastic and reliability analysis of a propeller with model reduction
}

\author{
Othmane Bendaou*,** — Jhojan Enrique Rojas**,*** \\ Abdelkhalak El Hami** — Abdeslam Aannaque* \\ Mohamed Agouzoul*
}

\author{
* Ecole Mohammadia d'Ingénieurs, Département Mécanique \\ UFR M.C.I.C.M., BP 765 Rabat-Agdal Maroc \\ mohamed.agouzoul@menara.ma \\ ** Institut National des Sciences Appliquées de Rouen \\ Laboratoire de Mécanique de Rouen BP 08, Avenue de l'Université \\ F-76801 Saint-Etienne du Rouvray cedex France aelhami@insa-rouen.fr \\ *** School of Mechanical Engineering, Federal University of Uberlândia \\ Uberlândia,Brézil,domingos@ufu.br
}

\begin{abstract}
Nowadays, design based on purely deterministic analyses has been replaced by stochastic and reliability analyses which consider the uncertainties affecting the design parameters. But from a numerical point of view, these analyses become costly for industrial mechanical applications (modelled by finite elements method) because of their great number of freedom degrees. In this work, we take an interest in reducing the CPU time for stochastic and reliability analyses of an industrial mechanical application by the modal condensation of his numerical model with the component mode synthesis method. The example of a propeller is studied to validate the proposed methods. The results of this study tend to show the considerable gain in CPU which we save by the using of our methodology.

RÉSUMÉ. De nos jours, la conception basée sur des analyses purement déterministes a été remplacée par des analyses stochastiques et fiabilistes qui prennent en considèration les incertitudes affectant les paramètres de conception. Mais d'un point de vue numérique, ces analyses deviennent coûteuses pour des applications mécaniques industrielles (modélisées par éléments finis) en raison de leur grand nombre de degrés de liberté. Dans ce travail, nous nous intéressons à la réduction du temps CPU pour des analyses stochastiques et de fiabilité d'une application mécanique industrielle par la condensation modale de son modèle numérique avec la méthode de synthèse modale. L'exemple d'une hélice est étudié pour valider les méthodes proposées. Les résultats de cette étude tendent à montrer le gain considérable en temps CPU que nous économisons par l'emploi de notre démarche.

KEYWORDS: modal analysis, stochastic analysis, perturbation, reliability analysis, heuristic based reliability method.

MOTS-CLÉS : analyse modale, analyse stochastique, perturbation, analyse fiabiliste, méthode d'optimisation heuristique.
\end{abstract}

DOI:10.3166/EJCM.18.195-215 @ 2009 Lavoisier, Paris 


\section{Introduction}

The dynamic analysis of industrial mechanical systems is often costly and sometimes difficult due to the computer resources limitations. Furthermore, these mechanical systems are often made of several parts, which for organization reasons, are calculated and tested independently by different teams. The sub-structuring methods constitute often the only resolution strategy. The use of these methods is then justified by the numerical benefit and by taking organization constraints of such big projects into account. One of the most used dynamic sub-structuring strategies is based on a component mode synthesis. In the reference (Craig, 1995) we find a synthesis of these methods. One of the pioneer works on reduction model of repetitive structures is presented in (ElHami et al., 1996).

One of the main hypothesises in the study of mechanical systems is that the model is deterministic. That means that the parameters used in the model are constant. However the experimental works show the limitations of such assumption. This is because there are always differences between what we calculate and what we measure due mainly to the uncertainties in geometry, the material properties, the boundary conditions or the load, which has a considerable impact on the vibrating behaviour of mechanical systems. This is why it is important to use numerical methods in order to take these uncertainties into count. The first methods were iterative (Shinozuka, 1972), whereas most recent are noniterarative. In (Impollonia et al., 2005; Kleiber et al., 1995; Muscolino et al., 1999; Van den Nieuwenhof et al., 2003; Dessombz, 2000) we find many noniterarative approaches to treat mechanical systems with uncertain parameters.

Nowadays, design based on purely deterministic analyses has been replaced by probabilistic-based reliability analyses which consider the uncertainties affecting the design parameters. In this context, reliability analysis intends to find the best compromise between cost and safety and to supply guidelines for carrying out reliable and cost-effective projects, accounting for the statistical variability of the design parameters. Many "efficient" methods have been devised as alternatives to Monte Carlo simulation. These methods include for example the first and second order reliability method respectively. These methods replace the original deterministic model with a computationally efficient analytical model in order to speed up the analysis (Haldar et al., 2000).

Regarding optimization, it is well known that the solution of reliability analysis problems by using classical methods, such as first and second order reliability method, is a difficult task due to the existence multiple most probable points of the limit state functions, mainly in the cases where a large number of design variables are involved (Der Kiureghian et al., 1991). First and second order reliability methods (FORM and SORM, respectively) are based on the gradient reliability methods which uses Newton-Raphson (N-R) procedure (Haldar et al., 2000). Aiming to eliminate these difficulties, this paper suggests using a modified approach based on first and second order reliability method (FORM and SORM) for the determination of reliability index 
and design points, based on ant colony optimization (ACO). In this context, an algorithm is proposed which is able to solve the global search optimization in reliability problems by using a Heuristic Based Reliability Method (HBRM) (Rojas et al., 2007). When compared with FORM and SORM methods, HBRM it's different because not requires the initial guess and the computation of gradients of the limit state function because it's based on multidirectional search. This algorithm is based on its ability to solve global optimization problems efficiently. The analysis methodology integrates a set of reliability analysis tools.

\section{Modal synthesis}

We suppose that we have a dynamical problem, divided into Ns sub-structure. In this paper, the exponents (and indices) "s" will designate the numbers of substructures. Each sub-structure occupies a volume noted $\Omega^{s}$. The interface $I^{s s^{\prime}}=\Omega^{s} \cap \Omega^{s^{\prime}}$ designates the junction between the sub-structure $\mathrm{s}$ and the substructure $\mathrm{s}^{\prime} . I^{s s^{\prime}}=\varnothing$ if the two domains are not in contact.

\subsection{Equations for the sub-structures}

It is assumed that each sub-structure is elastic, linear, isotropic and without any initial stress or strain. In the absence of voluminal source, the equation which models their vibratory behaviors is given by:

$$
\nabla \cdot \sigma^{s}-\rho_{s} u^{s}=0, \quad s=1, \ldots, N s
$$

$\rho_{s}, u^{s}$ et $\sigma^{s}$ are respectively the density, the displacement field and the constraints tensor of the sub-structure s.

If we note $\Gamma_{u}^{s}$ the borders of imposed displacement and $\Gamma_{f}^{s}$ the borders of imposed external effort, the boundary conditions associated to the substructure s are written:

$$
\left.u^{s}\right|_{\Gamma_{u}^{s}}=\bar{u}^{s},\left.\quad \sigma^{s} \cdot n\right|_{\Gamma_{f}^{s}}=\bar{f}^{s}
$$

In the sub-structure/sub-structure interfaces $\left(I^{s s^{\prime}}\right)$, the continuity of the displacements and the normal component of the constraints tensor must be assured. These conditions are written:

$$
\begin{aligned}
& \left(u^{s}-u^{s^{\prime}}\right)_{I^{s s^{\prime}}}=0 \\
& \left(\sigma^{s} . n-\sigma^{s^{\prime}} . n\right)_{I^{s s^{\prime}}}=0
\end{aligned}
$$




\subsection{Discretization by finite elements}

After application of the Finite Elements Method, we find the following algebraic form:

$$
\left[M^{s}\right]\left\{\ddot{u}^{s}\right\}+\left\{K^{s}\right\}\{u\}=\left\{f^{s}\right\}+\sum_{\substack{s^{\prime}=1 \\ s^{\prime} \neq s}}^{N s}\left\{f_{I}^{s s^{\prime}}\right\} \quad s=1, \ldots, N s
$$

The vector $\left\{u^{s}\right\}$ contains all the unknown degrees of freedom associated to the structural displacements of the sub-structure s (the known degrees of freedom on the border $\Gamma_{\mathrm{u}}^{\mathrm{s}}$, are not contained in this vector). $\left[M^{s}\right],\left[K^{s}\right]$ and $\left\{f^{s}\right\}$ respectively designate the mass matrix of the sub-structure $s$, its stiffness matrix and its vector of the external equivalent forces (including the inherent excitations of imposed displacements on the border $\Gamma_{u}^{s}$ ).

The term $\left\{f_{I}^{s s^{\prime}}\right\}$ physically represents the interfacial forces exerted on the substructure s by the adjacent sub-structure s'.

We assemble the Ns substructures according to a total vector containing the all degrees of freedom, organized according to the following form:

$$
\langle u\rangle=\left\langle\begin{array}{llll}
u^{1} & u^{2} & \ldots & u^{N s}
\end{array}\right\rangle
$$

The assembly of the formulation [5] leads to the following algebraic system:

$$
[M]\{\ddot{u}\}+[K]\{u\}=\{f\}+\left\{f_{I}\right\}
$$

where:

$$
\begin{aligned}
& {[M]=\left[\begin{array}{ccc}
M^{1} & & \\
& \ddots & \\
& & M^{N s}
\end{array}\right], \quad[K]=\left[\begin{array}{ccc}
K^{1} & & \\
& \ddots & \\
& & K^{N s}
\end{array}\right],} \\
& \{f\}=\left\{\begin{array}{c}
f^{1} \\
\vdots \\
f^{N s}
\end{array}\right\}, \quad\left\{f_{I}\right\}=\left\{\begin{array}{c}
\sum_{s^{\prime} \neq 1} f_{I}^{1 s^{\prime}} \\
\vdots \\
\sum_{s^{\prime} \neq N s} f_{I}^{N s s^{\prime}}
\end{array}\right\}
\end{aligned}
$$

\subsection{Calculation of local modes}

The vector of the freedom degrees of each sub-structure $\mathrm{s}$ is partitioned according to the intern freedom degrees (index i) and those of junctions (index $\mathrm{j}$ ). 
These latter correspond to the degrees of freedom situated at the interfaces between the sub-structure s and the all other adjacent sub-structures. Then, we write:

$$
\left\langle u^{s}\right\rangle=\left\langle u_{i}^{s} \mid u_{j}^{s}\right\rangle, \quad\left[M^{s}\right]=\left[\begin{array}{cc}
M_{i i}^{s} & M_{i j}^{s} \\
M_{j i}^{s} & M_{j j}^{s}
\end{array}\right], \quad\left[K^{s}\right]=\left[\begin{array}{cc}
K_{i i}^{s} & K_{i j}^{s} \\
K_{j i}^{s} & K_{j j}^{s}
\end{array}\right]
$$

According to the Craig and Bampton method, the chosen local modes correspond to the modes with fixed interfaces $I^{s s^{\prime}}$. They verify the flowing eigenvalues problem:

$$
\left\lfloor K_{i i}^{s}-\omega^{2} M_{i i}^{s}\left\{\left\{\psi_{i}^{s}\right\}=\{0\} \quad s=1, \ldots, N s\right.\right.
$$

These orthogonal modes are enriched by static modes of connection. These latter are defined as being the static deformation of the considered sub-structure, when a unit displacement is applied by turns to each of its junction degrees of freedom, the others being forced to 0 .

The local modal base of a sub-structure $\mathrm{s}$ is thus given by:

$$
\left[\varphi^{s}\right]=\left[\begin{array}{cc}
\Psi^{s} & -K_{i i}^{s^{-1}} K_{i j}^{s} \\
0 & I_{j j}^{s}
\end{array}\right]
$$

where $\left[\psi^{s}\right]$ represents the matrix of the modes with fixed interfaces retained after truncation and which are disposed in columns. The strategy of choice of the retained number of modes for each sub-structure consists in retaining the all modes contained in the multiple band of the useful frequency $\left(F_{u}\right)$, that is to say twice the useful band ([0 $\left.2 F_{u}\right]$ ) (El Hami et al., 1993).

The physical degrees of freedom of each substructure can then be decomposed on their respective local modal base:

$$
\left\{u^{s}\right\}=\left\lfloor\varphi^{s}\right\}\left\{\alpha^{s}\right\} \quad s=1, \ldots, N s
$$

$\left\{\alpha^{s}\right\}$ is the vector of the generalized coordinates associated to the sub-structure s, containing:

- the coefficients associated to the fixed interfaces modes;

- the junctions physical degrees of freedom.

\subsection{Model reduction}

The local decompositions [13] can be assembled, as follows:

$$
\{u\}=[\varphi]\{p\}
$$


where:

$$
[\varphi]=\left[\begin{array}{ccc}
\varphi^{1} & & 0 \\
& \ddots & \\
0 & & \varphi^{N s}
\end{array}\right] \quad \text { and } \quad\{p\}=\left\{\begin{array}{c}
\alpha 1 \\
\vdots \\
\alpha^{N s}
\end{array}\right\}
$$

Then, the equation [7] becomes after projection:

$$
\left[M_{p}\right]\{\ddot{p}\}+\left[K_{p}\right]\{p\}=\left\{f_{p}\right\}+[\varphi]^{t}\left\{f_{I}\right\}
$$

where:

$$
\left[M_{p}\right]=[\varphi]^{t}[M \rrbracket \varphi], \quad\left[K_{p}\right]=[\varphi]^{t}\left[K \rrbracket[\varphi], \quad\left[f_{p}\right]=[\varphi]^{t}[f]\right.
$$

Now, it is necessary to take the continuity conditions at the structure/structure interfaces into account. Indeed, the degrees of freedom of $\{p\}$ are not linearly independent. The linear relations between these degrees of freedom result from the equality of displacements at the structure/structure interfaces. They can be expressed by a connectivity global matrix [S]:

$$
\{p\}=[S\}\{q\}
$$

where $\{q\}$ contains only the linearly independent degrees of freedom. [S] characterizes the connectivity of the sub-structures between them. For the Craig and Bampton method, the matrix $[\mathrm{S}]$ is Boolean and easy to express since the junction physical degrees of freedom belong explicitly to the generalized unknown factors $\{\mathrm{p}\}$.

According to the conditions [4], there are the following equations of compatibility:

$$
\left\{f^{s s^{\prime}}\right\}+\left\{f^{s^{\prime} s}\right\}=\{0\}
$$

Then, we can show that these equations imply:

$$
[S]^{t}[\varphi]^{t}\left\{f_{I}\right\}=[S]^{t}\left\{f_{I}\right\}=0
$$

Thus, the final system to solve is written:

$$
\left\lfloor M_{q}\right\rfloor\{\ddot{q}\}+\left\lfloor K_{q}\right\rfloor\{q\}=\left\{f_{q}\right\}
$$

with:

$$
\left[M_{q}\right]=[S]^{t}\left[M_{p}\right][S], \quad\left[K_{q}\right]=[S]^{t}\left[K_{p}\right][S], \quad\left[f_{q}\right]=[\varphi]^{t}\left[f_{p}\right]
$$

Compared with the system [7], this model is considerably reduced since its size corresponds to the total number of orthogonal local modes retained after truncation, to which it is added the total number of junction degrees of freedom. 


\section{Stochastic study}

\subsection{Monte Carlo Simulation}

The estimation of the natural frequency moments (average and variance) of a structure could be obtained by the Monte Carlo simulation (Shinozuka, 1972). This is a very widely used method in spite of its high cost of calculation, and it's used as a reference for others approached calculation.

The Monte Carlo method has the advantage of taking into account all types of uncertainties on the parameters of a mechanical system. However, one of its main disadvantages is the CPU time needed because of its iterative nature.

\subsection{Perturbation methods}

The perturbation methods are very widely used in the stochastic finite elements domain. They are based on a development in Taylor series of the F.R.F. (respectively the eigen frequencies or the temporal response) in relation to the basis random physical variables, mechanical properties, geometrical characteristics or applied forces (the random parameters must appear explicitly in the dynamic matrices). The perturbation methods calculate the average and the standard deviation of the F.R.F. of a mechanical structure that has uncertain parameters. This method is used in many areas in order to solve linear and non-linear problems, for either static or dynamic modes.

The Muscolino perturbation method (Muscolino et al., 1999) could be used for a mechanical system whose the random parameters are independent. It is based on a development into a first-order Taylor series.

For a structure with uncertain parameters, it is assumed that the mass matrix and the stiffness matrix are functions of the random variables $\left\{\alpha_{p}\right\}_{(p=1, \ldots \ldots ., P)}$. And we note $\lambda_{i}$ the $i^{\text {th }}$ natural frequency.

The vector of the average parameters is defined by $\{\bar{\alpha}\}$, and the quantity d $\alpha$ is defined by $\{d \alpha\}=\{\alpha\}-\{\bar{\alpha}\}$.

The following notation is used to simplify the writing:

$$
[A]^{0}=[A]_{\{\bar{\alpha}\}}, \quad[A]^{n}=\left.\frac{\partial[A]}{\partial \alpha_{n}}\right|_{\{\bar{\alpha}\}}
$$

$[A]^{0}$ et $[A]^{n}$ are deterministic. The repetition of the indice " $\mathrm{n}$ " two times implies a summation. 
For a Muscolino perturbation method, we have:

$$
\begin{aligned}
& {[K]=[K]^{0}+[K]^{n}\left\{d \alpha_{n}\right\}} \\
& {[M]=[M]^{0}+[M]^{n}\left\{d \alpha_{n}\right\}} \\
& F=(F)^{0}+(F)^{n}\left\{d \alpha_{n}\right\}
\end{aligned}
$$

The eigen values-vectors equation of 0 order is written:

$$
\left([K]^{0}-\left(\lambda_{i}\right)^{0}[M]^{0}\right)\left\{\phi_{i}\right\}^{0}=0
$$

The eigen values-vectors equation of 1 order gives:

$$
\left(\lambda_{i}\right)^{n}=\frac{{ }^{t}\left\{\phi_{i}\right\}^{0}\left([K]^{n}-\left(\lambda_{i}\right)^{0}[M]^{n}\right)\left\{\phi_{i}\right\}^{0}}{{ }^{t}\left\{\phi_{i}\right\}^{0}\left([M]^{0}\right)\left\{\phi_{i}\right\}^{0}}
$$

The average is given by:

$$
E\left[\lambda_{i}\right]=\left(\lambda_{i}\right)^{0}
$$

The variance is given by:

$$
\operatorname{Var}\left[\lambda_{i}\right]=\left(\left(\lambda_{i}\right)^{n}\right)^{2} \operatorname{Var}\left(\alpha_{n}\right)
$$

If the Muscolino Perturbation Method is combined with a modal reduction method, it will lead to a substantial gain in CPU time. Nonetheless, this noniterative method has the limitation of not taking into account the geometry uncertainties which don't appear explicitly in the dynamic matrices.

\subsection{Perturbation methods with modal synthesis}

For a mechanical system whose dofs are reduced by the modal synthesis method, it is assumed that the modal bases are deterministic for the Muscolino perturbation method. This assumption is justified, since the perturbation method is only applied to systems, whose parameters vary weakly. Therefore:

$$
\begin{array}{ll}
{\left[M_{q}\right]^{0}=[S]^{t}[\varphi]^{t}[M]^{0}[\varphi][S]} & {\left[M_{q}\right]^{n}=[S]^{t}[\varphi]^{t}[M]^{n}[\varphi \rrbracket S]} \\
{\left[K_{q}\right]^{0}=[S]^{t}[\varphi]^{t}[K]^{0}[\varphi][S]} & {\left[K_{q}\right]^{n}=[S]^{t}[\varphi]^{t}[K]^{n}[\varphi][S]}
\end{array}
$$

\section{Reliability analysis}

Since the design parameters are considered as random variables, the satisfactory performance of a system can not be absolutely guaranteed. However, it can be 
expressed in terms of the probability of a certain failure criterion to be satisfied. In engineering terminology, this probability is called reliability and their counterpart, the fault probability. Thus, reliability is defined as the probability related to a perfect operation of a system (within the bounds specified by the design) during a predefined period in normal operation conditions.

Defining the design variables $X_{i}$ of the structure and a performance function expressed or limit state function as $Z=g\left(X_{i}\right)$ which delimits the surface of failure (defined by the condition $Z=0)$, the safe region $(Z>0)$ and unsafe region $(Z<0)$, of the design space in which the failure occurs, the failure probability is calculated as in (Haldar et al., 2000):

$$
P_{f}=\int_{g\left(X_{i}\right)<0} f_{X}\left(X_{i}\right) d x
$$

where $f_{X}\left(X_{i}\right)$ is the joint probability density function (PDF) of the design variables.

In practice, it is impossible to obtain the joint PDF in Equation [33] because of scarcity of statistical data. Even in the case where statistical information may be sufficient to determine these functions, it is often impractical to perform numerically in the integration indicated in Equation [33]. Moreover, the number of random variables is high; these variables do not appear explicitly in the performance function and there may be correlation among the design variables. These difficulties have motivated the development of various approximate reliability methods (Fiessler et al., 1979).

The main approaches to solve this equation are direct integration of PDF over the failure domain, analytical approximations such as the first and second order reliability methods (FORM and SORM, respectively). These methods use an optimization approach which is close to the methodology presented in this paper. Because of that, they are briefly reviewed in the following section.

\subsection{Reliability index estimation as a general optimization problem}

In traditional deterministic design optimization, the optimization problem is generally formulated in the physical space of the design variables and consists in minimizing or maximizing an objective function subjected to geometrical, physical or functional constraints in the form:

$$
\min f(\{y\})
$$

subjected to $g_{k}(\{y\}) \leq 0$, where $\{y\}$ designates the vector of deterministic design variables. 
In reliability analysis, which involves random variables $\{x\}$, the deterministic optimal solution is not considered the exact solution of the optimum design but is one of the most probably design. In this case, the failure surface or limit state function is given by $G(\{x\},\{y\})=0$. This surface defines the limit between the safe region $G(\{x\},\{y\})>0$ and unsafe region of the design space. The failure occurs when $G(\{x\},\{y\})<0$, and the failure probability is calculated as $P_{f}=\operatorname{prob}[G(\{x\},\{y\}) \leq 0]$.

The reliability index $\beta$ is introduced as a measure of the reliability level of the system and is estimated in the so-called reduced coordinate system, where the random variables $\{u\}$ are statistically independent with zero mean and unit standard deviation.

Thus a pseudo-probabilistic transformation $\{u\}=T[\{x\},\{y\}]$ must be defined for mapping the original space into the reduced coordinate system (Wang et al., 1996). Considering that the probability density in the reduced space decays exponentially with the distance from the origin of this space, the point with maximum probability of failure (most probable point) on the limit state surface is the point of minimum distance from the origin. The reliability index is thus defined as the minimum distance between the origin of the reduced space and the hyper surface representing the limit state function $H(\{u\},\{y\})$. Hence, it is possible to find the most probable point or design point by solving a constrained optimization problem that is:

$$
\beta=\min \sqrt{\sum_{i=1}^{n} u_{i}^{2}}
$$

subjected to safety constraints: $H(\{u\},\{y\})=0$ By formally introducing a cumulative density function $(\Phi)$ of the normal probability distribution function, the first order approximation (tangent plane at the MPP) to $P_{f}$ can be written as (Haldar et al., 2000):

$$
P_{f}=\Phi(-\beta)
$$

This corresponds to the substitution of the hyper surface by the hyper plane passing through the point defined by $u_{i}$.

\subsection{Assessment of first and second order reliability methods}

FORM and SORM can be considered as gradient-based methods since they demand the evaluation of the partial derivatives of the limit state function with respect to the random variables at each iteration step. 
FORM is based on linear (first order) approximation of the limit state surface tangent to the most probable point of the failure surface to the origin of a reduced coordinate system. Thus, the random variables are transformed to reduced variables in a reduced coordinate system. For estimating the reliability index based on FORM one can use the algorithm suggested by (Rackwitz et al., 1978) in which the limit state function does not need to be solved because a Newton-Raphson type recursive algorithm is introduced to find the design point (Der Kiureghian et al., 1991). This algorithm has been widely used in the literature (Haldar et al., 2000).

SORM estimates the probability of failure by using a nonlinear approximation of the limit state function by a second order representation. The curvatures of the limit state function are approximated by the second-order derivatives with respect to the original variables. Thus, SORM improves FORM by including additional information about the curvature of the limit state function through of a curvature parameter. SORM was explored by (Fiessler et al., 1979) using quadratic approximations. In this work the authors use a simple closed-form solution for the computation of failure probability using a second-order approach given by (Breitung, 1984) based on the theory of asymptotic approximation.

It is important notice that the most probable point of FORM and SORM is the same. Additionally, SORM uses as initial value the reliability index value estimated through FORM. Zhao and Ono (Zhao et al., 1999) and Rojas (Rojas et al., 2006) give more details about these classical techniques.

\subsection{Reliability assessment by heuristic based method}

The solution of the optimization problem given by Equation [35] by using classical gradient-based optimization methods is not a simple task due to the existence of local minima in the design space and the necessity of computation of the gradients (partial derivatives). As result, accuracy, convergence and computational effort are relevant issues. The existence of multiple MPPs is similar to multiple local minima in optimization. The solutions of many problems in structural optimization can be considered to be satisfactory once a local minimum is reached. However, this is an unacceptable procedure in reliability analysis since the local MPP may not represent the worst failure scenario and the actual failure may occur below the predicted level. Hence, only the global MPP represents the actual structural reliability (Wang et al., 1996).

Another difficulty that must be remembered is that traditional methods FORM and SORM require an initial guess of the solution (reliability index and random variables) and it is not always possible to assure global convergence. These aspects has motivated the authors of this paper to explore an alternative approach for estimation of reliability index, which do not require the computation of gradients of the limit state function and are intrinsically based on multidirectional search. In this work the authors use the approach that uses Finite Element analysis to evaluate implicit limit state functions and is based on a HBRM, which allows the use of optimization methods such as Genetic Algorithms (GA) (Michalewicz, 1994) and (Haupt et al., 2004)), Particle Swarm Optimization (PSO) (Kennedy et al., 1995) 
and Ant Colony Optimization (ACO) (Venter et al., 2002). It is believed that such approach can circumvent some of the difficulties mentioned above, and thus can lead to improved results of reliability analysis.

Figure 1 shows a scheme of HBRM algorithm when each heuristic method has stop criteria. The parameters $\lambda, \gamma$ and $\mathrm{C}$ are the penalty factors used in the optimization procedure. $G\left(x_{i}\right), \mu_{x_{i}} \sigma_{x_{i}}$ and $x_{i}^{\prime}$ represent the limit state function, mean, standard deviation and design variables in the reduced coordinate system, respectively. (Rojas et al, 2007) and (Rojas, 2008) give more details of HBRM.

Taking into account the performance of HBRM, it was observed that this methodology is able to handle multiple limit state functions based on numerical models and probabilistic variables related to geometrical, load and material properties parameters. Rojas (Rojas et al., 2007) give more details of HBRM specifically the numerical applications use the ACO. The following section discusses the main ideas about this heuristic technique.

A scheme of the reliability analysis methodology that couples reliability tools (FORM, SORM and HBRM) with the finite element models of a structure is shown in Figure 2. In the reliability procedure it is possible to choose a reliability method. The evaluation of the limit state function is made through an interface between mechanical and reliability procedures. In this work the structural responses used in the reliability analysis are the natural frequencies of a structure.

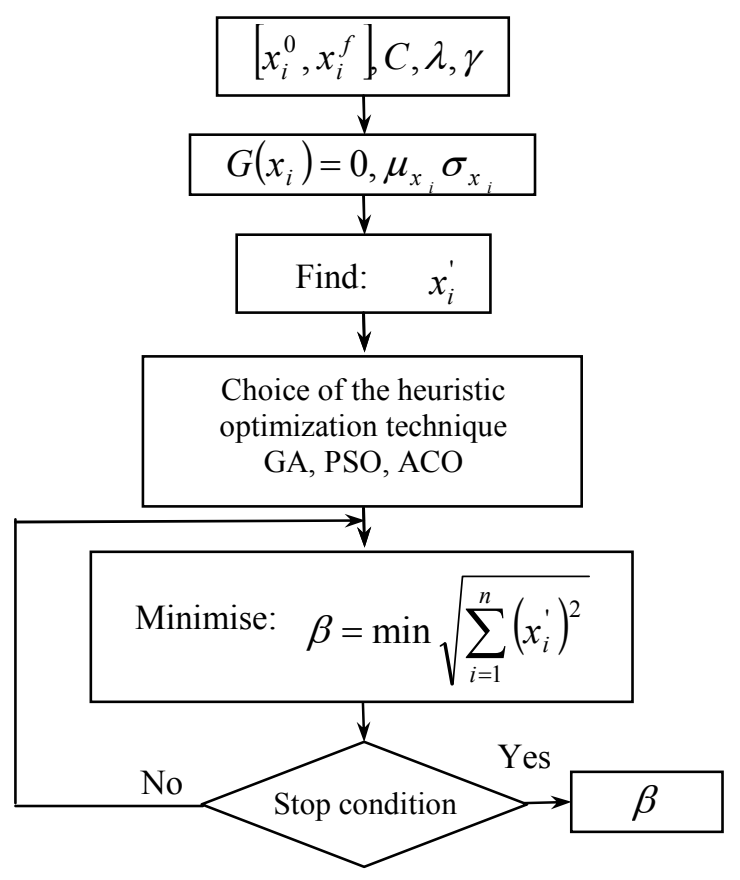

Figure 1. HBRM algorithm 


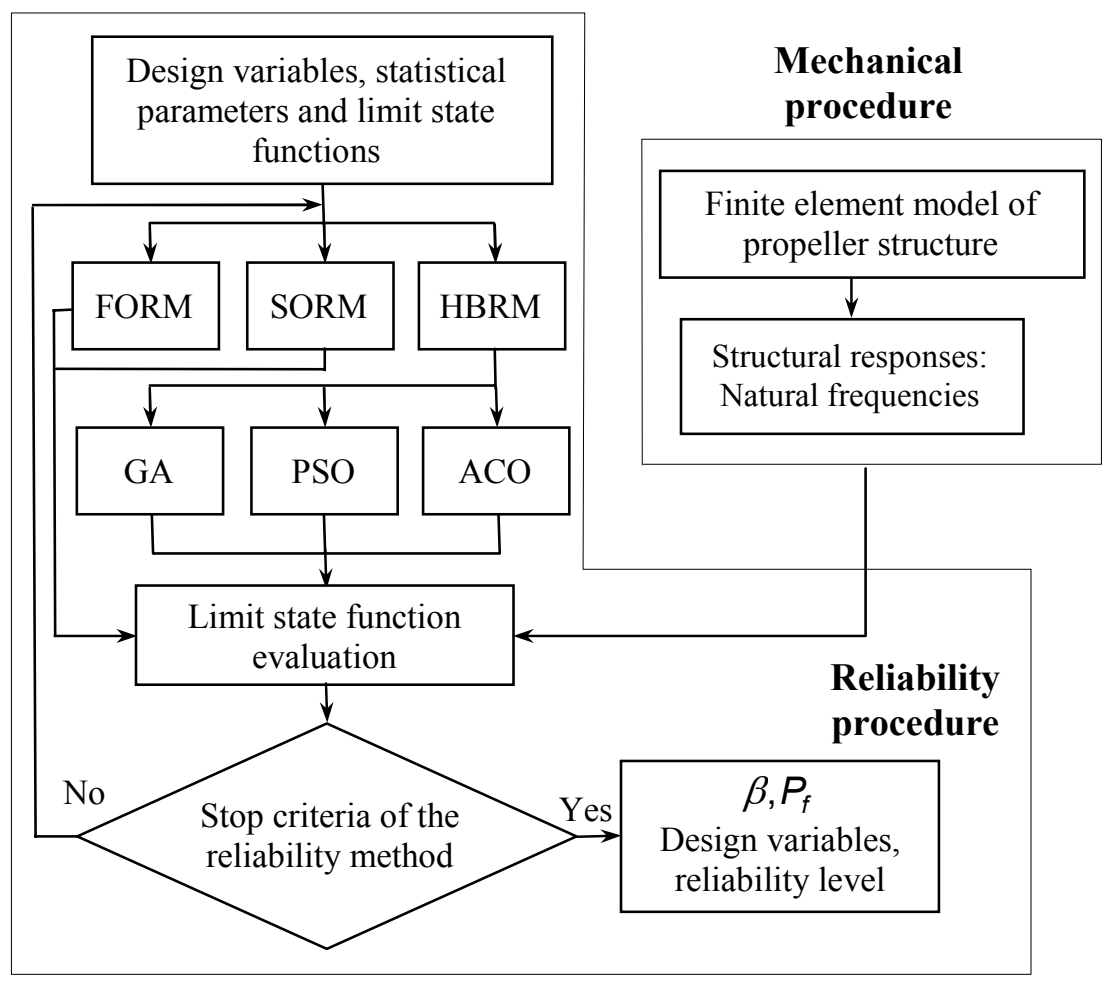

Figure 2. Reliability analysis methodology

Numerical applications for HBRM use the ACO. The following section discusses the main ideas about this heuristic technique.

\subsection{Ant colony optimization}

ACO, introduced by Marco Dorigo in his doctoral thesis in 1992 (Dorigo, 1992), is a probabilistic technique for solving computational problems, which can be reduced to find good paths through graphs. This technique follows some basic concepts, as presented below (Socha et al., 2004):

- search performed by a population of ants, i.e., by simple independent agents in a incremental construction of solutions;

- probabilistic choice of solution components based on stigmergic information of pheromone. A stigmergic process is the process through which the results of a worker insects activity acts as a stimulus to further activities;

- no direct communication between ants. 
ACO is inspired by the behavior of real ants and their communication scheme by using pheromone trails (Dorigo, 1992). A moving ant lays some pheromone on the ground, thus marking the path. The collective behavior that emerges from the participating agents is a form of positive feedback where the greater the number of ants that follow a trail, the more attractive that trail becomes. When searching for food, real ants start moving randomly, and upon finding food they return to their colony while laying down pheromone trails. This means that, if other ants find such a path, they return and reinforce it. However, over time the pheromone trail starts to evaporate, thus reducing its attractive strength. When a short and a long path are compared, it is easy to see that a short path gets marched over faster and thus the pheromone density remains high. Thus, if one ant finds a short path (from the optimization point of view, it means a good solution) when marching from the colony to a food source, other ants are more likely to follow that path, and positive feedback eventually encourages all the ants to follow the same single path.

The idea behind ACO is to mimic this behavior by using artificial ants. The outline of a basic ACO algorithm is as follows:

1. Define the ACO parameters (trail weight, initial trail value, etc.).

2. Create an initial colony (it just allocates memory).

3. Put the entire colony in the nest.

4. For all ants do a complete tour (go from nest to food).

5. Update the pheromone trail.

6. Go to step 3 and repeat until the stop criteria is achieved.

Details about how ACO is implemented can be obtained from Dorigo, 1992), (Socha et al., 2004) and (Pourtakdoust et al., 2004).

\section{Industrial application}

In order to validate the suggested methods, we studied the dynamic behavior of a boat propeller. The geometrical model of this propeller (Figure 3) was designed by means of "SolidWorks" and "Ansys". The mesh as well as the geometrical substructuring (Figure 4) was carried out with "Ansys". whereas the processor part was carried out on the one hand with "Ansys" for deterministic calculation without dofs reduction (the result of this calculation will be regarded as reference), and on the other hand with codes elaborated with "Matlab" (on the basis of the mesh carried out with "Ansys") for deterministic, stochastic and reliability analysis, with and without dofs reduction.

The mesh was made with tetrahedral elements. For calculation with modal synthesis (dofs reduction) we divided the propeller into four sub-structures. 

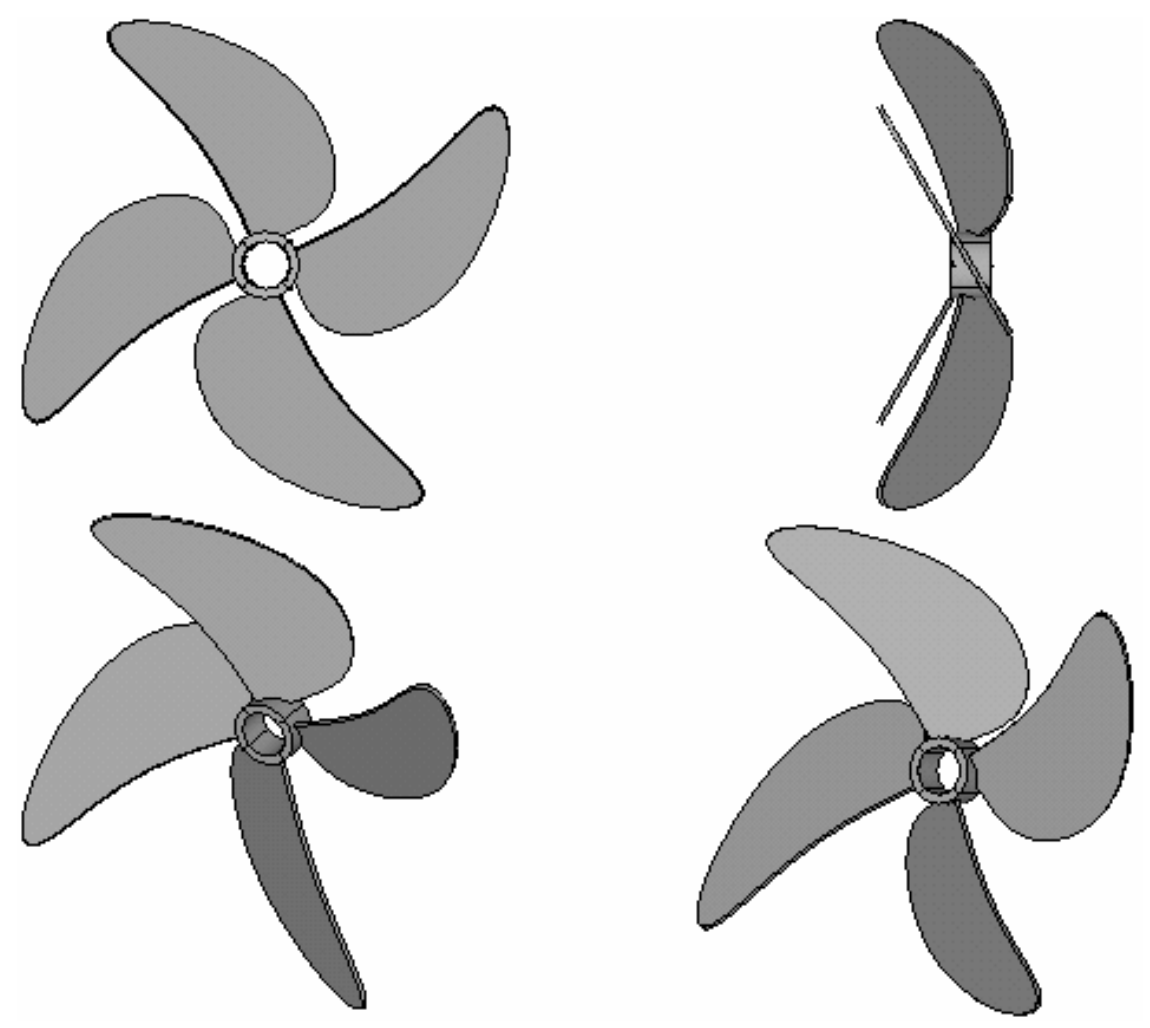

Figure 3. Boat propeller
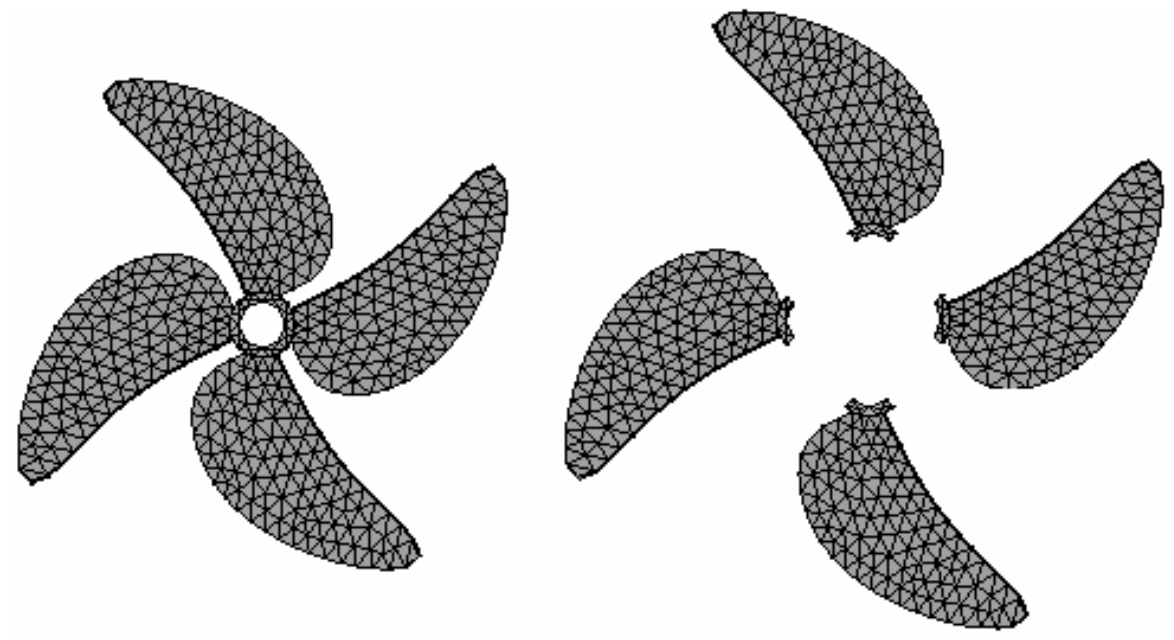

Figure 4. Mesh and sub-structuring 


\subsection{Deterministic modal Analysis}

In the Table 1, the modal analysis of the propeller is exposed, and its six first natural frequencies calculed with:

- « Ansys » without dofs reduction (Reference) ;

- «Matlab » without dofs reduction;

- «Matlab» with dofs reduction (synthesis modal).

are compared. The Calculations are made by supposing that the material parameters are fixed:

$$
E=2.1 e^{11}[\mathrm{~Pa}], \quad \rho=7860\left[\mathrm{Kg} / \mathrm{m}^{3}\right] \text { and } \quad v=0.3
$$

Table 1. The six first natural frequencies of the propeller (and the errors in relation to the reference solution)

\begin{tabular}{cccccc} 
& \multicolumn{3}{c}{$\begin{array}{c}\text { Direct } \\
\text { calculation }\end{array}$} & \multicolumn{2}{c}{$\begin{array}{c}\text { Calculation with Modal } \\
\text { Synthesis }\end{array}$} \\
\hline mode & with Ansys (reference) & \multicolumn{2}{c}{ with Matlab } & \multicolumn{2}{c}{ with Matlab } \\
\hline 1 & 100 & 100 & $(0.0 \%)$ & 100 & $(0.0 \%)$ \\
2 & 295 & 295 & $(0.0 \%)$ & 295 & $(0.0 \%)$ \\
3 & 346 & 346 & $(0.0 \%)$ & 346 & $(0.0 \%)$ \\
4 & 915 & 914 & $(0.1 \%)$ & 914 & $(0.1 \%)$ \\
5 & 1225 & 1224 & $(0.1 \%)$ & 1224 & $(0.1 \%)$ \\
6 & 1519 & 1518 & $(0.1 \%)$ & 1518 & $(0.1 \%)$ \\
\hline \multicolumn{5}{c}{2286 dofs }
\end{tabular}

The time that calculation with « Matlab» required:

$\begin{array}{lll}\text { - for direct calculation } & : & 6 \mathrm{~min} 30 \mathrm{~s} \\ \text { - for calculation with modal synthesis } & : & 62 \mathrm{~ms}\end{array}$

This result shows us how much we save in time CPU, when we carry out a model reduction by modal synthesis.

\subsection{Stochastic modal analysis}

After the deterministic modal analysis, we passed to the modal analysis in the case where the Young modulus and the density of the structure are normal random variables which we know the averages and the standard deviations: 
Table 2. Random variables and statistics parameters of the propeller

\begin{tabular}{cccc}
\cline { 2 - 4 } & Distribution & Mean & Standard deviation \\
\hline$E(\mathrm{~Pa}) \times \mathrm{e}^{11}$ & normal & 2.1 & 0.021 \\
$\rho\left(\mathrm{Kg} / \mathrm{m}^{3}\right)$ & normal & 7860 & 78.60 \\
\hline
\end{tabular}

Computer codes were elaborate with «Matlab» to calculate the moments (averages and standard deviations) of the first 6 natural frequencies of the propeller, with 3 different methodologies:

- Procedure 1 (classical procedure): direct calculation with Monte Carlo simulation (for 100 samples;

- Procedure 2: direct calculation with Muscolino perturbation method;

- Procedure 3 (proposed procedure): modal synthesis with Muscolino perturbation method.

Let us note that "Procedure 1" is a classical Procedure which we will use as reference.

Table 3. The means of the six first natural frequencies of the propeller (and the errors in relation to the reference solution)

\begin{tabular}{cccccc} 
& $\begin{array}{c}\text { Procedure 1 (reference) } \\
\text { mode }\end{array}$ & \multicolumn{2}{c}{ Procedure 2 } & \multicolumn{2}{c}{$\begin{array}{c}\text { Procedure 3 } \\
\text { - Classical procedure - }\end{array}$} \\
\hline 1 & 100 & 100 & $(0.0 \%)$ & 100 & $(0.0 \%)$ \\
2 & 295 & 295 & $(0.0 \%)$ & 295 & $(0.0 \%)$ \\
3 & 346 & 346 & $(0.0 \%)$ & 346 & $(0.0 \%)$ \\
4 & 914 & 914 & $(0.0 \%)$ & 914 & $(0.0 \%)$ \\
5 & 1224 & 1224 & $(0.0 \%)$ & 1224 & $(0.0 \%)$ \\
6 & 1519 & 1518 & $(0.1 \%)$ & 1518 & $(0.1 \%)$ \\
\hline
\end{tabular}

Table 4. The standard deviation of the six first natural frequencies of the propeller (and the errors in relation to the reference solution)

\begin{tabular}{cccccc} 
& $\begin{array}{c}\text { Procedure 1 (reference) } \\
\text { mode }\end{array}$ & \multicolumn{2}{c}{ Procedure 2 } & \multicolumn{2}{c}{$\begin{array}{c}\text { Procedure } 3 \\
\text { - Proposed procedure - }\end{array}$} \\
\hline 1 & 12 & 12 & $(0.0 \%)$ & 12 & $(0.0 \%)$ \\
2 & 35 & 35 & $(0.0 \%)$ & 35 & $(0.0 \%)$ \\
3 & 41 & 41 & $(0.0 \%)$ & 41 & $(0.0 \%)$ \\
4 & 109 & 109 & $(0.0 \%)$ & 109 & $(0.0 \%)$ \\
5 & 146 & 145 & $(0.7 \%)$ & 145 & $(0.7 \%)$ \\
6 & 181 & 180 & $(0.5 \%)$ & 180 & $(0.5 \%)$ \\
\hline
\end{tabular}

The time that the calculation of the 6 first natural frequencies moments of the propeller with « Matlab » required: 


$\begin{array}{lll}- \text { Procedure 1 } & : & 17 \mathrm{~h} 36 \mathrm{~min} \\ \text { - Procedure 2 } & : & 26 \mathrm{~min} \mathrm{30 \textrm {s }} \\ \text { - Procedure 3 } & : & 234 \mathrm{~ms}\end{array}$

The propeller example shows that the saving in CPU time is very important, when we dynamically analyze a structure with uncertain parameters by the modal synthesis method allied to the Muscolino perturbation method.

\subsection{Reliability calculation}

The reliability analysis methodology (illustrate in Figure 2) used in this numerical application integrates a set of reliability analysis tools (based on FORM, SORM and HBRM) developed under MATLAB $^{\circledR}$ with finite element analysis using the commercial software ANSYS ${ }^{\circledR}$. In the numerical applications, the reliability analysis takes into account the design parameters considered in deterministic and stochastic analysis. Table 2 summarizes the design parameters and their statistical moments considered in this example. The study of reliability analysis is based on a single state limit function which considers the first natural frequency $\left(f_{1}\right)$ of the propeller, such as:

$$
G(E, \rho)=1-\frac{f_{1}}{f_{\lim }}, \text { where } f_{\lim }=98 \mathrm{~Hz}
$$

Table 5 summarizes the results obtained by Newton-Raphson in FORM and SORM approaches and ACO in HBRM. The limit state function is evaluated by FEM method. The Table 5 and Table 6 shows the reliability analysis which is carried out with in without modal reduction:

Table 5. Reliability results without modal reduction

\begin{tabular}{cccc} 
& FORM & SORM & HBRM (LIT) \\
\hline$E(P a) \times \mathrm{e}^{11}$ & 2.16 & 2.16 & 2.13 \\
$\rho\left(\mathrm{kg} / \mathrm{m}^{3}\right)$ & 7580.5 & 7580.5 & 7571.7 \\
$\beta$ & 3.8745 & 3.8747 & 3.33 \\
Pf $(\%)$ & $5.34 \mathrm{e}^{-3}$ & $5.34 \mathrm{e}^{-3}$ & 0.043 \\
Reliability & 99.99 & 99.99 & 99.96 \\
$(\%)$ & Time(FORM) +Time(SORM) \\
\hline \multicolumn{3}{c}{ 138.6 } \\
Time (min) & Time(FORM) < Time(SORM) & 230.1 \\
\hline
\end{tabular}

FORM and SORM results depend strongly on the initial guess and its CPU time it is very similar because SORM is based on FORM results for improve the first order approaches. It is important to remember that FORM results are initial solution for SORM algorithm. Therefore, in this application, SORM can not improve FORM approximations. 
Table 6. Reliability results with modal reduction

\begin{tabular}{cccc} 
& FORM & SORM & HBRM (LIT) \\
\hline$E(P a) \times \mathrm{e}^{11}$ & 2.16 & 2.16 & 2.13 \\
$\rho\left(\mathrm{kg} / \mathrm{m}^{3}\right)$ & 7580.5 & 7580.5 & 7571.7 \\
$\beta$ & 3.8745 & 3.8747 & 3.33 \\
Pf $(\%)$ & $5.34 \mathrm{e}^{-3}$ & $5.34 \mathrm{e}^{-3}$ & 0.043 \\
Reliability & 99.99 & 99.99 & 99.96 \\
$(\%)$ & Time(FORM) +Time(SORM) & 34.9 \\
\hline \multicolumn{3}{c}{21.5} \\
Time (min) & Time(FORM) < Time(SORM) \\
\hline
\end{tabular}

When compared to FORM and SORM, HBRM is advantageous since it requires neither the initial guess nor the computation of gradients of the limit state function and must powerful to find global minimums. Indeed, several studies in the literature show that heuristic methods are more efficient than methods based in the calculation of gradients regarding the calculation of global minimums.

The obtained results exhibit that the predicted reliability levels using HBRM are accurate in comparison with similar approaches that uses FORM and SORM to evaluate implicit limit state functions. The results obtained show the potentialities of HBRM despite the large time CPU compared with FORM and SORM counterparts.

Numerical comparison between FORM, SORM and HBRM showed in the preceded tables demonstrate that reliability results are satisfied for all approaches. Modal synthesis combined to classical methods and HBRM have a good performance. Considering its characteristics, we remark that the HBRM has a better performance in relation to classical reliability techniques despite a small time differences.

Reliability study with methods combined to the modal synthesis method leads to a very important gain in CPU time. Where the interest to carry out a reliability analysis by the HBRM with modal reduction.

\section{Conclusion}

In order to be able to make stochastic and reliability analysis for large structure dynamical problems with a reasonable computing time, we proposed to combine the modal synthesis method (sub-structuring method) with the Muscolino Perturbation method for stochastic analysis and with the HBRM for reliability analysis. The proposed methodology were applied to a propeller whose the Young modulus and the density are random variables. The results are very hopeful and tend to show the effectiveness of our methodology. 


\section{References}

Breitung K., "Asymtotic Approximation for Multinormal Integrals", Journal of Engineering Mechanics, ASCE, vol. 110, n 3, 1984, p. 357-366.

Craig R.R., "Substructure methods in vibration, Transactions of the ASME", Journal of Vibration and Acoustics, vol. 117, 1995, p. 207-213.

Der Kiureghian A., De Stefano M., "Efficient algorithm for second-order reliability analysis", Journal of the Engineering Mechanics Division, American Society of Civil Engineers, vol. $117, \mathrm{n}^{\circ} 12,1991$.

Dessombz O., Analyse des structures comportant des paramètres incertains, Thèse de doctorat, Ecole Centrale de Lyon, France, 2000.

Dorigo M., Optimization, Learning and Natural Algorithms, Ph.D. Dissertation, Politecnico di Milano, Italy, 1992.

El Hami A., Lallement G., Minotti P., Belhadj H., Cogan S., "Methods combining finite group theory with component modes synthsis in the analysis of repetitive structures", Computer and Structures, vol. 48, $\mathrm{n}^{\circ}$ 6, 1993, p. 975-982.

El Hami A., Radi B., "Some Decomposition Methods in the Analysis of repetitive structures", Computers \& structures, vol. 58, n 5 , 1996, p. 973-980.

Fiessler B., Neumann H.J., Rackwitz R., "Quadratic Limit States in Structural Reliability", Journal of Engineering Mechanics, ASCE, vol. 1095, n 4, 1979, p. 661-676.

Haldar A., Mahadevan S., Reliability Assessment Using Stochastic Finite Element Analysis, Ed. John Wiley \& Sons, Inc., New York, USA, 2000.

Haupt R.L., Haupt S.E., Pratical Genetic Algorithms, Wiley-Interscience Publication, New York, USA, 2004.

Impollonia N., Ricciardi G., "Explicit solutions in the stochastic dynamics of structural systems", Probabilistic Engineering Mechanics, vol. 21, 2005, p. 171-181.

Kennedy J., Eberhart R.C., "Particle Swarm Optimization", Proceedings of the 1995 IEEE International Conference on Neural Networks, Perth, Australia, 1995, p. 1942-1948.

Kleiber M., Hien T.D., The stochastic finite element method, Ed. Jhon Wiley, 1992.

Michalewicz Z., Genetic Algorithms + Data Structures = Evolution Programs, SpringerVerlag, New York, USA, 1994.

Mohsine A., Contribution à l'optimisation Fiabiliste en Dynamique des Structures Mécaniques. Thèse de Doctorat, Institut National des Sciences Appliquées de Rouen, France, 2006.

Muscolino G., Ricciardi G., Impollonia N., "Improved dynamic analysis of structures with mechanical uncertainties under deterministic input", Probabilistic Engineering Mechanics, vol. 15, 1999, p. 199-212.

Pourtakdoust S.H., Nobahari H., "An Extension of Ant Colony to Continous Optimization Problems", Dorigo, M. (ed.), Proceedings of the ANTS 2004 - Fourth International Workshop on Ant Colony Optimization and Swarm Intelligence, Brussels, Belgium, 2004. 
Rackwitz R., Fiessler B., "Structural Reliability Under Combined Random Load Sequences", Computers and Structures, vol. 9, $\mathrm{n}^{\circ}$ 5, 1978, p. 484-494.

Rojas J.E., Viana F.A., Rade D., Steffen Jr. V., "A Procedure for Structural Reliability Analysis Based on Finite Element Modeling", IV Congresso Nacional de Engenharia Mecânica, 22 a 25 de Agosto 2006, Recife-PE, Brasil.

Rojas J.E., Viana F.A., Rade D., "Natured-inspired Optimization Techniques Applied to Structural Reliability Analysis", $7^{\text {th }}$ Multidisciplinary International Conference Quality and Reliability (RAMS), 20-22 March 2007, Tangier, Morocco.

Rojas J.E., Reliability Analysis Methodology based on Heuristic Optimization Techniques and Meshless Methods Applied to Mechanical Systems (in French), Doctoral Thesis, Institut National des Sciences Appliquées de Rouen, France, 2008.

Shinozuka M., "Monte Carlo solution of structural dynamics", International Journal Computer Structural, vol. 2, 1972, p. 855-874.

Socha K., "ACO for Continuous and Mixed-Variable Optimization”, Dorigo, M. (ed.), Proceedings of the ANTS 2004 - Fourth International Workshop on Ant Colony Optimization and Swarm Intelligence, Brussels, Belgium, 2004.

Van den Nieuwenhof B., Coyette J.P., "Modal approaches for the stochastic finite element analysis of structures with material and geometric uncertainties", Computers Methods in applied mechanics and engineering, vol. 192, 2003, p. 3705-3729.

Vanderplats G.N., Numerical Optimization Techniques for Engineering Design, Vanderplaats Research and Development, Inc., $3^{\text {rd }}$ edition, 1999.

Venter G., Sobieski J.S., "Multidisciplinary Optimization of a Transport Aircraft Wing using Particle Swarm Optimization", Proceedings of the $9^{\text {th }}$ AIAA/ISSMO Symposium on Multidisciplinary Analysis and Optimization, vol. AIAA 2002-5644, Atlanta, GA, September 4-6, 2002.

Wang L., Grandhi R.V., "Safety Index Calculation Using Intervening Variables for Structural Reliability Analysis", Computers \& Structures, vol. 59, n 6, 1996, p. 1139-1148.

Zhao Y.G., Ono T., "A general Procedure for First/Second-order Reliability Method (FORM/SORM)”, Structural Safety, vol. 21, 1999, p. 95-112.

Received: 5 September 2007

Accepted: 6 October 2008 
